\title{
Age of diagnosis-based linkage analysis in type 1 diabetes
}

\author{
Andrew D Paterson and Arturas Petronis
}

Neurogenetics Section, Clarke Division, Centre for Addiction and Mental Health, Toronto, Ontario, Canada

\begin{abstract}
Genetic linkage studies of type 1 diabetes have produced a number of conflicting results, suggesting a high degree of locus heterogeneity in this disease. Approaches which model such heterogeneity will increase the power to fine map susceptibility loci. Here, using data from a genome scan of 356 affected sib pairs with type 1 diabetes, we performed heterogeneity analysis based on similarity of age at diagnosis of the sib pairs. We observed linkage to the region on chromosome 4p16.3 in sib pairs both diagnosed over the age of 10 years, whilst there was no evidence for linkage in sib pairs diagnosed before age 10 years. In contrast the sib pairs diagnosed before the age of 10 years demonstrated linkage to IDDM 10, on chromosome 10p. Age of diagnosis-based heterogeneity analyses in complex diseases may be particularly helpful in mapping some susceptibility loci. European Journal of Human Genetics (2000) 8, 145-148.
\end{abstract}

Keywords: type1 diabetes; linkage; genome scan; age of onset; Huntington's disease; Wolfram syndrome

\section{Introduction}

Cloning genes for complex diseases is proving to be a difficult task and this is probably due to the absence of major genes, low penetrance of susceptibility alleles on the phenotypes studied, and a high degree of genetic locus heterogeneity. Attempts to reduce the degree of locus heterogeneity using the criterion of age of diagnosis (AOD) has been helpful in the mapping and identification of a number of human disease genes. These include: BRCAl (No.113705 ${ }^{1}$ ) and BRCA2 (No. $600185^{1}$ ), responsible for a proportion of early onset breast cancer; APP, PSEN1 and PSEN2 in early onset Alzheimer's disease (No. 104760; No. 104311; No. 600759 respectively ${ }^{1}$ ); SNCA in Parkinson disease (No. 163890 ${ }^{1}$ ) among others. Typel diabetes AOD may be in part genetically determined. ${ }^{2}$ Molecular studies support age-dependent effects for both HLA genotypes and various biomarkers in type 1 diabetes, with strongest associations general ly in early onset cases (No. 222100, ${ }^{*} 138275^{1}$ ). Age-dependent linkage of diabetes to Idd4 has been shown in the NOD mouse model. ${ }^{3}$ We hypothesized that using families with affected individuals of similar AOD may be more homogeneous genetically than unselected families, and may show linkage

Correspondence: Andrew Paterson, Neurogenetics Section, Clarke Division, Centre for Addiction and Mental Health, 250 College Street, Toronto, Ontario, M5T 1R8, Canada. Tel: +1 4165358501 X 4421; Fax: +1 416979 4666; E-mail: andrew.paterson@utoronto.ca Received 12 July 1999; revised 5 October 1999; accepted 11 November 1999 to particular chromosomal regions, and have tested this using data from a large genome scan for type 1 diabetes.

\section{Methods}

Pedigree and genotyping data for 351 genome-wide markers for 356 affected sib-pair families from the UK were used (obtained from http://diesel.cimr.cam.ac.uk/todd/). These families were recruited by the British Diabetes Association ${ }^{4}$ and include 93families from the original 'UK96' genome scan, ${ }^{5}$ as well as 263 additional families. ${ }^{6}$ AOD data were provided by Charles Mein. The AOD distribution in the siblings from these families are presented in Figure 1 , and is bimodally distributed. This reflects the AOD ascertainment scheme used for this study whereby the majority of families recruited had one sibling with AOD $<17$ years of age and the other $<29$ years. ${ }^{5,6}$ The mean age of diagnosis is 10.3 years (median 10.0years). Since the mean and median AOD in this data were both close to age 10 years, the families were divided into those where both sibs were affected earlier or at 10years ( $n=130$ sib pairs, henceforth termed $E$ ) and those where both pairs had onset later than 10 years ( $n=95 \mathrm{sib}$ pairs, termed L). This left 131 families in neither group which were not analysed further. Multipoint linkage analysis was performed separately in the $E$ and $L$ groups using the MAPMAKER SIBS v 2.0 program $^{7}$ under the assumption of no dominance variance, reporting the maximum multipoint lod score (MMLS). Alleles had been scored uniquely in each 


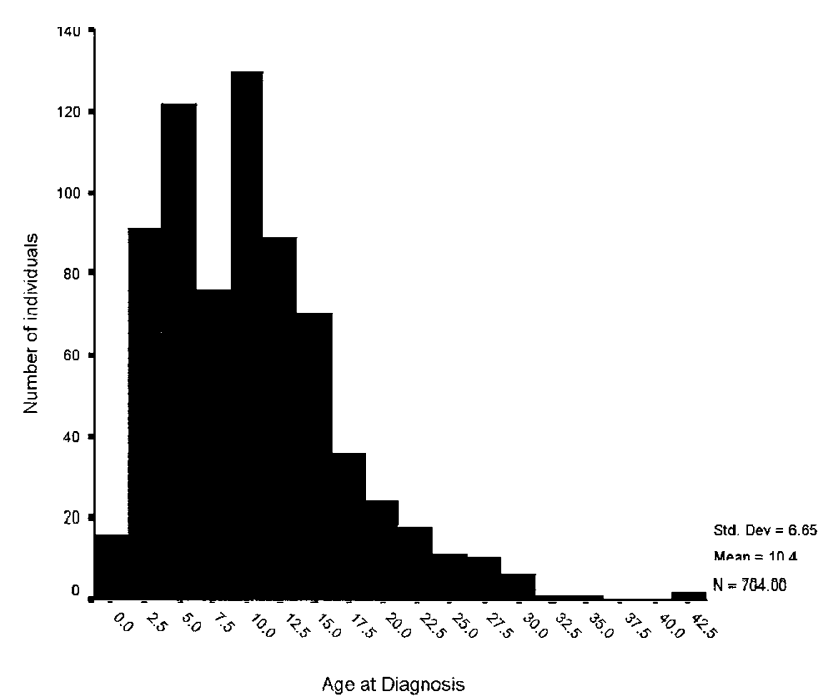

Figure 1 Age of onset distribution in 356IDDM affected sib pairs; each sibling was treated as a separate datum. Mean age of onset $=10.3$ years, and median $=10.0$ years.

family using a 4-allele system, with the alleles observed in each family numbered 1-4. Since estimates of allele frequencies are required for linkage analysis, we used $1 / n$ for the frequency of each allele at a marker with $n$ al leles. The marker order and sex-averaged inter-marker recombination fractions provided by Dr Todd's web site were used.

\section{Results}

Only regions which demonstrate MMLS > 2.0 are reported here. At the HLA region of chromosome $6 p$, evidence for linkage derived from both early and later onset sib pairs as

a

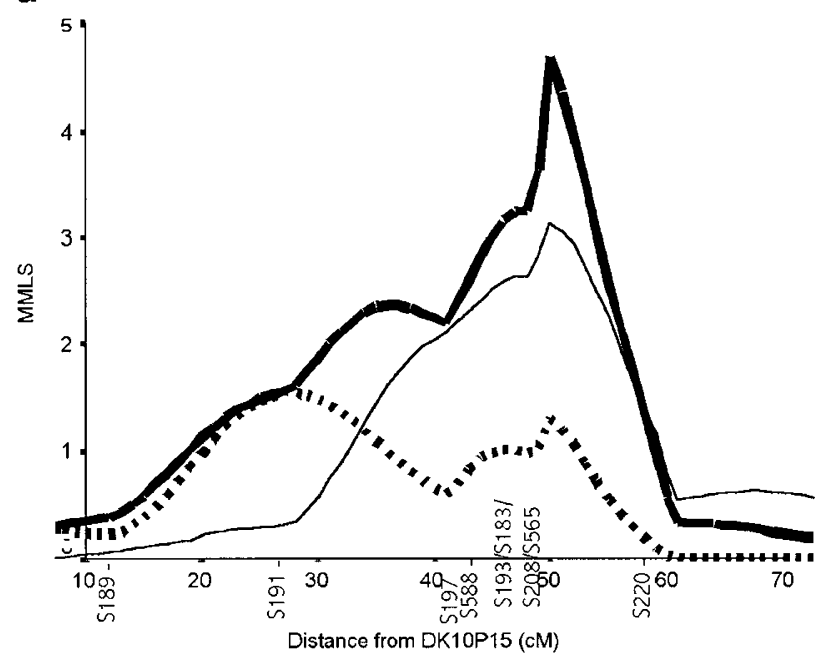

follows: $M M L S=13.1$ for the $E$ group and $M M L S=8.10$ from the $L$ group. No difference in the MMLS at HLA between the two groups was seen when the number of families in each group was taken into account. In addition, there was evidence for linkage to chromosome10 in the region where IDDM 10 has been mapped., 5,8 The peak linkage at this region occurs around markers D10S193-D10S183D10S208-D10S565 and arises predominantly from the E group (MMLS = 3.2 for $E$ compared with $M M L S=1.2$ for $L$; Figure2a). The only other region which produces M MLS $>2$ was on the tip of chromosome $4 p$, where there was evidence for linkage in the L sib pairs close to D4S412 (MMLS = 2.13; Figure 2b). At the peak of linkage to the $L$ group there was no evidence for linkage to the $E$ group (MMLS $=0.25$ ), but interestingly, there was evidence of linkage in the early onset families more proximally between D4S2366 and D4S431 (MMLS $=1.90)$, where the $L$ families show much weaker linkage (MMLS $=0.52$ ). No MMLS $>2.0$ was observed in either $E$ or $L$ families at the human region that is homologous to mouse Idd4.

Because alleles were not scored consistently across families, we assessed whether the results on chromosomes 4 and 10 were dependent on specification of allele frequencies, and used the sib_ibd option of ASPEX v $2.1^{9}$ to determine sharing identical by descent (Table1). At D4S412 there are nominally significant differences in allele sharing between $E$ and $L$ groups-at the other markers the differences are not significant, but they do support the findings of the multipoint analyses (Table1).

\section{Discussion}

This exploratory analysis has not been corrected for multiple statistical comparisons. In the primary analysis of this data

b

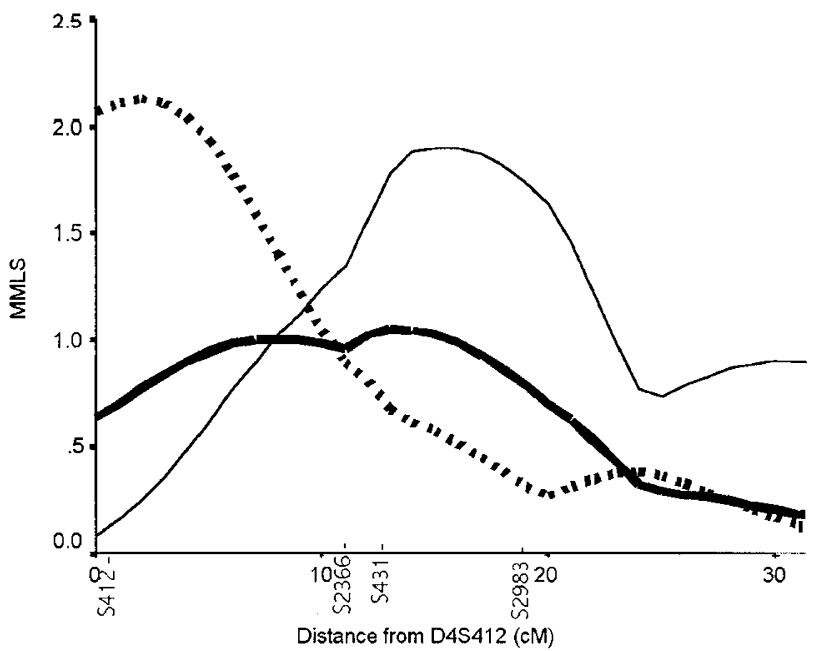

Figure 2 MMLS scores plotted for IDDM against distance in CM from the most pter marker for chromosome 10 (A) and chromosome 4 (B): Heavy solid line: all families; thin solid line: families with onset in both sibs $\leq 10$ years of age (E); heavy dotted line: families with onset in both sibs > 10 years (L). Approximate positions of markers on the X axis. D10S.. for A, D4S... for B. 
Table 1 Identity by descent sharing in early onset and late onset families at selected markers on chromosomes 4 and 10

\begin{tabular}{|c|c|c|c|c|c|c|c|c|c|c|c|c|c|}
\hline \multirow{2}{*}{$\begin{array}{l}\text { Distance } \\
\text { (cM) }\end{array}$} & \multirow[t]{2}{*}{ Marker } & \multicolumn{5}{|c|}{ Early onset } & \multicolumn{5}{|c|}{ Late onset } & \multicolumn{2}{|c|}{ Difference } \\
\hline & & IBD1 & IBDO & $\%$ IBD & $\chi^{2}$ & $P$ & |BD1 & IBD0 & $\%$ IBD & $\chi^{2}$ & $P$ & $\chi^{2}$ & $\mathrm{P}$ \\
\hline & D4S412 & 91 & 81 & 53 & 0.6 & 0.44 & 71 & 39 & 64 & 9.3 & 0.0023 & 3.7 & 0.05 \\
\hline 11.1 & D4S2366 & 95 & 78 & 55 & 1.7 & 0.19 & 68 & 51 & 57 & 2.4 & 0.12 & 0.14 & 0.71 \\
\hline 2.2 & D4S431 & 88 & 61 & 59 & 4.9 & 0.027 & 58 & 51 & 53 & 0.4 & 0.53 & 0.87 & 0.35 \\
\hline 6.5 & D4S2983 & 113 & 83 & 58 & 4.6 & 0.032 & 69 & 69 & 50 & 0.0 & 1.0 & 1.9 & 0.17 \\
\hline & D10S588 & 65 & 33 & 66 & 10.4 & 0.0013 & 41 & 35 & 54 & 0.5 & 0.48 & 2.8 & 0.097 \\
\hline 4.7 & D10S193 & 95 & 68 & 58 & 4.5 & 0.034 & 59 & 49 & 55 & 0.9 & 0.34 & 0.35 & 0.55 \\
\hline 1.0 & D10S183 & 100 & 57 & 64 & 11.8 & 0.00059 & 66 & 41 & 62 & 5.8 & 0.016 & 0.11 & 0.74 \\
\hline
\end{tabular}

IBD1: number of sib pairs sharing 1 allele identical by descent; IBD0: number of sib pairs sharing 0 alleles identical by descent; \%IBD: proportion of alleles shared identical by descent; $\chi^{2}$ and $P$ value assuming 1df; Difference: $2 \times 2$ contingency table for difference in sharing of alleles in early and late onset sib pairs; $\chi^{2}$ and P value assuming 1df. Distance: inter-marker distance according to data from the map provided at Dr Todd's web site.

18loci were reported which met the authors' criteria for linkage, ${ }^{6}$ whilst here we report only three chromosomal regions that meet our significance criteria. For the results obtained for chromosome 4 several additional reasons make these regions worthy of further investigation and these are outlined in the following.

The linkage results for the $E$ and $L$ groups on chromosome $4 p 16$ are supported by the observation that two diseases that have been mapped to this region exhibit an increased rate of diabetes. Specifically, evidence for linkage of L IDDM sib pairs to the tip of chromosome $4 p$ is of interest since D4S412 is only $2 \mathrm{cM}$ away from the gene responsible for Huntington's disease (HD) in the sex-averaged genetic map. ${ }^{10}$ Two studies have reported an increased incidence of abnormal glucose tolerance tests in HD patients. ${ }^{11,12}$ Furthermore, the prevalence of diabetes in a sample of 288 individuals with HD was higher than that in an age-matched representative sample of the US white population, ${ }^{13}$ with the mean AOD of diabetes in HD around 35-40 years. ${ }^{13}$ Animal studies further support the role of HD in etiology of diabetes. R6/2 mice which have a transgene containing about 140 CAG repeats in part of the human HD gene develop an insulin-responsive diabetes by age 7 weeks, ${ }^{14}$ and the pancreata, among other tissues, demonstrate intranuclear inclusions. ${ }^{15}$ Another gene closely linked to D4S412 is LRPAP1 (low density lipoprotein receptor-related protein-associated protein 1). LRPAP1 is expressed in human pancreas, and has been shown to contain a binding site for immune deposit-inducing antibodies which are a feature of an experimental autoimmune rat model of human membraneous glomerulonephritis, called passive Heymann nephritis. ${ }^{16,17}$ The above findings suggest that DNA sequence variation in or near either HD or LRPAP1 is a susceptibility factor for later onset type 1 diabetes.

The second locus on 4p16.1, between D4S2366 and D4S431 ( $E M M L S=1.90$ ), is of interest through it's putative association with the gene for Wolfram syndrome $\left(\right.$ No. $\left.222300^{1}\right)$. Wolfram syndrome is an autosomal recessive disorder, featuring juvenile onset diabetes with a mean onset of 6 years. The gene for Wolfram syndrome (W FS1) maps to the same bacterial artificial chromosome as D4S431. The risk of diabetes in siblings of Wolfram syndrome patients is about $10 \%{ }^{18,19}$ W FS1 has recently been shown to be expressed in pancreatic islet cells $\mathrm{s}^{20,21}$ making it a strong positional candidate for susceptibility to E-onset IDDM. Since the frequency of mutant WFS1 alleles may be about $0.3-1 \%$ further studies are necessary to clarify whether WFS heterozygotes are at increased risk for diabetes.

Evidence for linkage to a locus on chromosome10p13q11, termed IDDM10, has previously been provided. ${ }^{5,6,8}$ However, an other group has found only very weak linkage to IDDM 10 (MMLS $=0.4$ at D10S193 ${ }^{22}$ ) although some families which they studied overlap with those analysed here. The discrepancy between the results of the two studies could be due to different proportions of $E$ and $L$ families in each sample. Although AOD appears helpful in reducing locus heterogeneity in complex diseases, different age cut-offs likely apply at individual loci. Much larger sample sizes than analysed here will be necessary to clarify whether our results are type 1 errors, and to define AOD distributions at particular IDDM loci. AOD-based heterogeneity tests may assist in the fine mapping and ultimate cloning of susceptibility loci for complex diseases.

\section{Acknowledgements}

We thank Dr Todd and Charles Mein for making the data available, Cathy Spegg for assistance and Richard Van Holst for proof reading. ADP is a Fellow of the Medical Research Council of Canada, AP is an OMHF New Investigator and NARSAD Young Investigator.

\section{References}

1 Online Mendelian Inheritance in Man, OMIM (TM), Center for Medical Genetics, Johns Hopkins University (Baltimore, MD) and National Center for Biotechnology Information, National Library of Medicine (Bethesda, MD), 1999: http://www.ncbi.nlm.nih.gov/ omim/

2 Fava D, Gardner S, Pyke D, Leslie RD: Evidence that the age at diagnosis of IDDM is genetically determined. Diabetes Care 1998; 21: 925-929.

3 Ghosh S, Palmer SM, Rodrigues NR et al: Polygenic control of autoimmune diabetes in nonobese diabetic mice. Nat Genet 1993; 4: 404-409.

4 Bain SC, Todd JA, Barnett AH: The British Diabetic Association, Warren repository. Autoimmunity 1990; 7: 83-85.

5 Davies JL, Kawaguchi Y, Bennett ST et al: A genome-wide search for human type1 diabetes susceptibility genes. Nature 1994; 371: 130-136. 
6 Mein CA, Esposito L, Dunn MG et al: A search for type1 diabetes susceptibility genes in families from the United Kingdom. Nat Genet 1998; 19: 297-300.

7 Kruglyak L, Lander ES: Complete multipoint sib-pair analysis of qualitative and quantitative traits. Am J Hum Genet 1995; 57: 439-454.

8 Reed P, Cucca F, Jenkins $S$ et al: Evidence for a typel diabetes susceptibility locus (IDDM 10) on human chromosome10p11q11. Hum Mol Genet 1997; 6: 1011-1016.

9 Hinds DA, Risch N: The ASPEX package: affected sib-pair exclusion mapping, 1996. ftp://lahmed.stanford.edu/pub/aspex/

10 Collins A, Frezal J, Teague J, Morton NE: A metric map of humans: 23,500 loci in 850 bands. Proc Natl Acad Sci USA 1996; 93: 14771-14775; http://cedar.genetics.soton.ac.uk/public - html/

11 Podolsky S, Leopold NA, Sax DS: Increased frequency of diabetes mellitus in patients with Huntington's chorea. Lancet 1972; 1: 1356-1358.

12 Podolsky S, Leopold NA: Abnormal glucosetolerance and arginine tolerance tests in Huntington's disease. Gerontology 1977; 23: 55-63.

13 Farrer LA: Diabetes mellitus in Huntington disease. Clin Genet 1985; 27: 62-67.

14 Hurlbert MS, Zhou W, Wasmeier C, Kaddis FG, Hutton JC, Freed CR: Mice transgenic for an expanded CAG repeat in the Huntington's disease gene develop diabetes. Diabetes 1999; 48: 649-651.

15 Sathasivam K, Hobbs C, Turmaine M et al: Formation of polyglutamine inclusions in non-CNS tissue. Hum Mol Genet 1999; 8: 813-22.
16 Nielsen PR, Ellgaard L, Etzerodt M, Thogersen HC, Poulsen FM: The solution structure of the $\mathrm{N}$-terminal domain of alpha2macroglobulin receptor-associated protein. Proc Natl Acad Sci USA 1997; 94: 7521-7525.

17 Kerjaschki D, Ullrich R, Exner M, Orlando RA, Farquhar MG: Induction of passive Heymann nephritis with antibodies specific for a synthetic peptide derived from the receptor-associated protein. I Exp Med 1996; 183: 2007-2015.

18 Fraser FC, Gunn T: Diabetes mellitus, diabetes insipidus, and optic atrophy. An autosomal recessive syndrome? J Med Genet 1977; 14: 190-193.

19 Barrett TG, Bundey SE, Macleod AF: Neurodegeneration and diabetes: UK nationwide study of Wolfram (DIDMOAD) syndrome. Lancet 1995; 346: 1458-1463.

20 Inoue $\mathrm{H}$, Tanizawa $\mathrm{Y}$, Wasson J et al: A gene encoding a transmembrane protein is mutated in patients with diabetes mellitus and optic atrophy (Wolfram syndrome). Nat Genet 1998; 20: $143-148$.

21 Strom TM, Hortnagel K, Hofmann S et al: Diabetes insipidus, diabetes mellitus, optic atrophy and deafness (DIDMOAD) caused by mutations in a novel gene (wolframin) coding for a predicted transmembrane protein. Hum Mol Genet 1998; 7: 2021-2028.

22 Concannon P, Gogolin-Ewens KJ, Hinds DA et al: A secondgeneration screen of the human genome for susceptibility to insulin-dependent diabetes mellitus. Nat Genet 1998; 19: 292-296. 